\title{
GOAL-ORIENTED ADJUSTMENT MECHANISMS FOR STABILIZATION AND DEVELOPMENT OF TRANSITION ECONOMIES
}

\author{
Vasak Toroyan \\ Head of Department of Mathematical Modeling of Economy \\ Yerevan State University \\ vasaktoroyan@yahoo.com \\ Rolan Mnatsakanyan \\ Advisor to the Chairman of Central Bank \\ Republic of Armenia \\ 06cba@cba.am
}

Keywords: goal program, structural graph, co-integration, alternative version

\begin{abstract}
Summary: The method of goal program implementation lies in the basis of Economy Stabilization and development problem solution. Main objects for this program are distinguished. Possible versions are developed for each of them and feasible versions are constructed on this basis. Correlations in the program are considered on the basis of co-integration, which was implemented on the example of Central Bank.
\end{abstract}

\section{Introduction}

Macroeconomic policy aimed at stabilization and economic growth in transition economies has been implemented in the framework of structural changes, transition to market relations, institutional changes, new system of management and decision-making. Main directions of transition period macroeconomic policy include, particularly, structural, fiscal, monetary, foreign exchange, foreign financial and external debt problems and development problems of banking system, which represent an integral hierarchic program system. The development of this system is affected both by internal and external factors, their impact on all levels of economy and financial sectors is expressed simultaneously in different amount and lags. That's why it is necessary to form alternative versions for the development of this hierarchic system and to choose the best version among them based on some concept. These problems are discussed in the framework of Armenia Economy Stabilization and Development Goal Program (ESD GP), which represents a balanced set of structural, monetary, fiscal policy measures, that are aimed to provide sustainable growth of economic and social systems in short-term and long-term and achieve predetermined goal macro-indicators.

\section{The Structure of Goal Program Model}

Decision agreement in ESD GP has difficulties, as corresponding program measures are formed in different management systems of economic and financial sectors. In particular, it is necessary to coordinate some functions of Central Bank (CB) and Ministry of Finance to ensure efficient realization of monetary and fiscal policies. The problem is getting more difficult, when it is necessary to combine program measures of stabilization and the policy oriented on economic growth. In addition, as decisions about the development of different subsystems are worked out separately, they can disagree with each other and in fact the influence of internal and external factors in different situations can make necessary the coordination of decisions. In this case the problem is to interconnect feasible versions of different subsystems and construct feasible and compatible versions for one joint program based on this. 
For the construction of ESD GP following general principles are defined. First, ESD GP should have clear purposefulness, which should correspond with the goals of social-economic development and ensure precise interconnections of these goals. Second, it should include all subsystems and functional relationships aimed to achieve chosen goals. In accordance with this following stages are distinguished in the process of ESD GP formation: develop goal macroeconomic indicators and define program structure taking into account real and financial sectors of economy, as well as internal and external relationships; provide balanced financial flows and construct alternative versions based on this; select efficient version among alternative versions.

ESD GP includes foreign sector of economy, presented by balance of payments, monetary sector, presented by $\mathrm{CB}$, relationships between economy and financial sectors, presented by banking system, state budget, foreign currency, money, goods and labor markets. The diagram presenting all these connections is given in Figure 1.

Figure 1. The Structure of Economy Stabilization and Development Goal Program

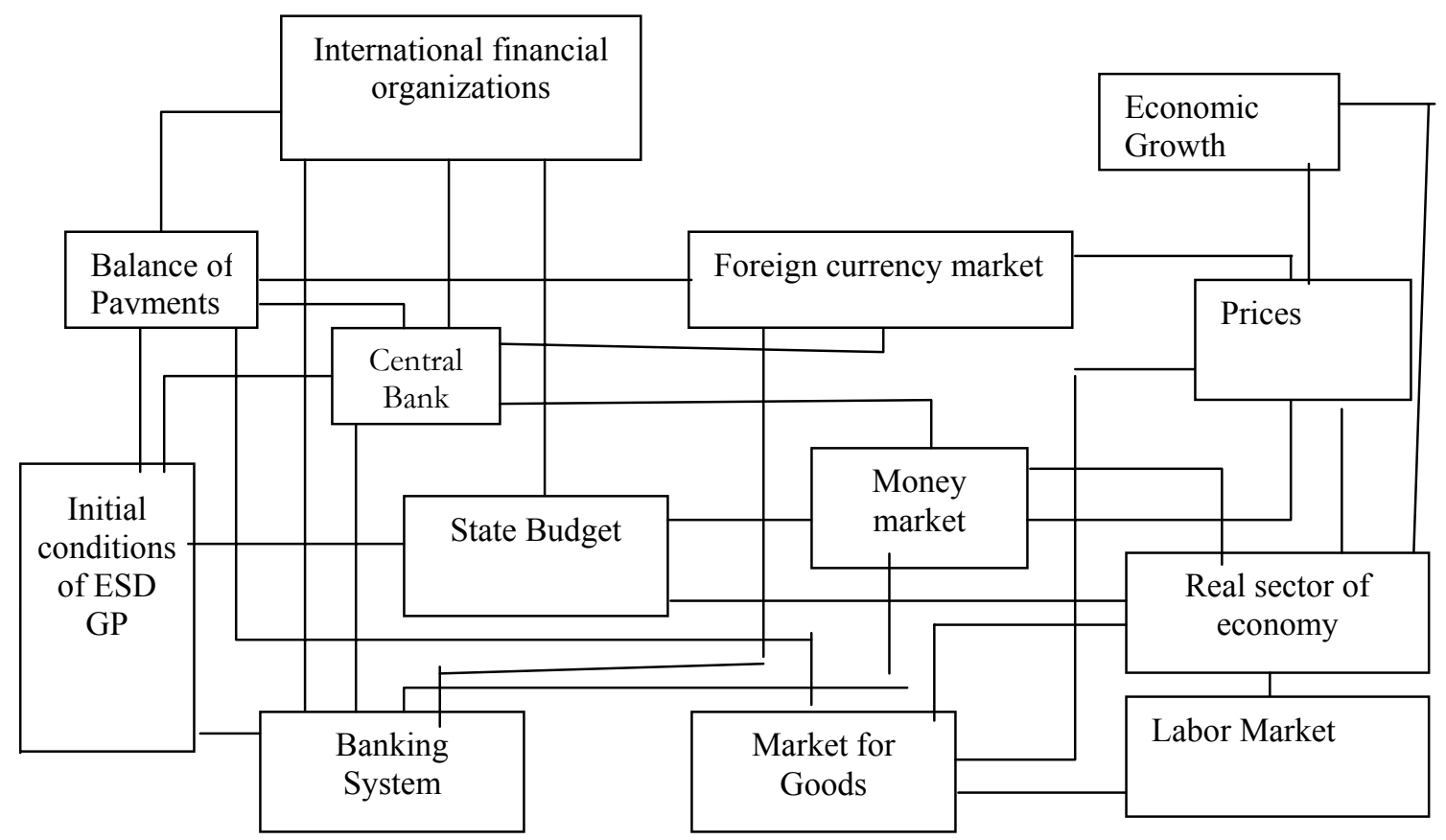

The monetarist approach for balance of payments and neoclassic model of economic growth is taken as a theoretical basis for ESD GP model. This makes possible combination of short-term financial stabilization with long-term economic growth. In the presence of significant structural problems and high level of unemployment in transition countries in accordance with our approach the expansion of money supply is increasing GDP, which means any change in demand brings to appropriate change in GDP. Under these conditions efficient implementation of monetary policy, i.e. adequate definition of REPO interest rate, level of domestic loans, monetary base and foreign currency exchange rate has a significant role.

The monetary policy may essentially affect expansion of the total demand through instruments of supply, however it often may become a real source, giving rise to economic imbalance and instability. In view of this, possibilities of using supply-oriented instruments of the monetary policy need to be enhanced and broadened in stabilization and development programs for countries in transition, implementation mechanisms will need to be brought in line with the real sector development and selected macro- 
indicators. The monetary policy instruments which are aimed at expanding or reducing the monetary base will need to be considered in view of increasing the GDP in both short term and medium term periods. This approach will make the economic stabilization program more lasting, which might positively affect the current account of Balance of Payments and internal equilibrium of the economy. Therefore, such an approach will enable to combine directions of the economic stabilization with structural policy that would considerably improve effectiveness of implemented programs.

Internal and external factors affecting ESD GP include influences from sharp changes in: a) foreign exchange resources from foreign direct investments and international organizations to finance the state budget deficit and regulate the current account, b) terms of trade, c) total domestic supply, and d) demand for money. These factors create sharp changes both in short term and medium term periods. Changes in the Capital Account lead to alteration of volumes of foreign direct investments. The level of using production capacities significantly affects the level of GDP. Changes in the monetary terms may reduce or expand demand for money and changes in the terms of trade may bring to higher or lower import costs in foreign currency.

To respond to mentioned internal and external factors, parameters such as REPO interest rate, monetary base, exchange rate and volumes of total domestic loans, have been chosen in ESD GP. Decisions made on them are based upon the following indicators of economic growth and excess demand: differences in nominal and maximum GDP growth rates, current account, inflation and the reaction of interest rates in financial market, and their dynamics. Alternative versions for the policy conduct are developed on the basis of various combinations of these four parameters of management. These include the rules of decision-making on combinations of REPO interest rates, monetary base, exchange rate and level and volumes of total domestic loans in the economy. Impact of alternative versions of the policy conduct upon other macro-indicators is expressed through the implementation of changes in Balance of Payments, GDP and the prices.

In the result of the influence of these four factors an alternative version of ESD GP is formed in the framework of each version of policy conduct. In fact, alternative versions include possible changes in macro-indicators, with their subsequent deviations, attributable to impact of internal and external shortand medium term factors. In the latter case, changes incurred by macro-indicators due to constantly changing macroeconomic environment, are considered.

ESD GP is most adequately presented through a structural graph. Theoretical concepts of macroeconomic, fiscal and monetary policies concerning relationships between spheres covered in the graph, and statistical estimates, based on econometric surveys, have served as a basis for determining the links between the vertices of the graph. Each vertex of the structural graph represents a separate subprogram, which, in its turn, reflects the possible scenarios or alternatives for development of certain economic or financial sector. Edges of the graph reflect causal relationships and financial flows between them. To get alternative versions for an appropriate detailed graph is constructed and all feasible scenarios for ESD GP satisfying financial and non-financial constraints of the model are determined based on this.

\section{The Model of the Program and Separation of Feasible Combinations}

Suppose $G=(X, \Gamma)$ is an oriented graph and as always $X \neq \varnothing, \Gamma$ is the reflection of $X$ in $X ; G$ will be called a structural graph. Each $x \in X$ is a set itself, so $X$ is a family of sets. Besides $x \neq \varnothing$ for every $x \in X, x_{1} \cap x_{2}=\varnothing$, if $x_{1} \neq x_{2}, x_{1}, x_{2} \in X$, let's denote $\Sigma=\bigcup_{x \in X} x$. Suppose $\Delta$ is the reflection of $\Sigma$ in $\Sigma$, so that the graph $H=(\Sigma, \Delta)$ is given, which we will call detailed with regard to $G$, if in addition three other conditions are satisfied (Toroyan and Aharonyan, 1997). 
The structural graph is given in the Figure 1, where the graph vertices are the sub-programs of ESD GP. Main macroeconomic indicators characterizing the given vertex were selected to construct projects for each of them. It is necessary to point out that some of these characterizing indicators may recur in a number of vertices. This proves that the vertices of the program are not strongly separated objects, but they are closely interrelated. The linkages of the structural and detailed graphs' vertices are drawn based on the statistic data analysis.

The separation of the projects' feasible combinations is a recurrent process, where the transfer from one graph to another occurs as following. An elementary cycle should be distinguished and separated in the structural graph. Then feasible combinations of this cycle should be separated and after that a new vertex of structural graph, projects of which are the feasible combinations of separated elementary cycle, should replace the elementary cycle. The links of the new vertex with the remaining vertices of the structural graph may be restored based on the following formulas.

Suppose $G=\left(x_{1}, \ldots, x_{n} ; \Gamma\right)$ and $G_{p}$ is a subgraph corresponding with some subprogram. All feasible combinations of projects for $G_{p}$ are selected with the help of elementary cycles. To be specific let's suppose that the vertices of $G_{p}$ are $x_{1}, \ldots, x_{k}$. Let's pass to a new graph $\bar{G}=\left(\bar{x}_{p}, x_{k+1}, \ldots, x_{n} ; \bar{\Gamma}\right)$, in which $G_{p}$ subgraph of $G$ graph is modified into a vertex $\bar{x}_{p}$ of graph $\bar{G} . \bar{\Gamma}$ is defined in the following way:

a) $\bar{\Gamma} \bar{x}_{p}= \begin{cases}\bigcup_{i=1}^{k} \Gamma x_{i} \cup \bar{x}_{p} \backslash \bigcup_{i=1}^{k} x_{i}, & \text { only in that case, when } \exists \mathrm{x}_{\mathrm{i}}, \mathrm{x}_{\mathrm{j}}, \mathrm{i}, \mathrm{j}=\overline{1, \mathrm{k}} \text { so that } \Gamma \mathrm{x}_{\mathrm{j}} \supset \mathrm{x}_{\mathrm{j}}, \\ \bigcup_{i=1}^{k} \Gamma x_{i} \backslash \bigcup_{i=1}^{k} x_{i}, & \text { in other cases. }\end{cases}$

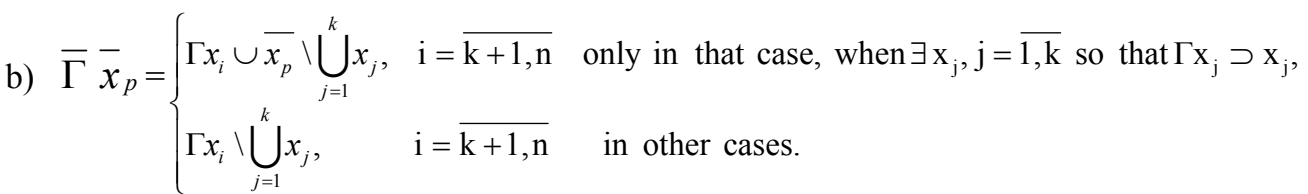

Accordingly the links of the detailed graph vertex may be restored by these formulas:

Suppose that the elements of the set $x_{i}, i=\overline{1, n}$ are ranged. Then we'll use $\Sigma_{i}=\left(\xi_{1}^{i}, \ldots, \xi_{n_{i}}^{i}\right), i=\overline{1, n}$ denotation instead of $x_{i}$, where $\xi_{r}^{i}, i=\overline{1, n}, r=\overline{1, n_{i}}$, is the $r$-th project of the $i$-th object.

Connections in $H=\left(\bar{\Sigma}_{p}, \Sigma_{k+1}, \ldots, \Sigma_{n} ; \bar{\Delta}\right)$ graph, which is the detailed graph of $\bar{G}$, are restored like in structural graph. The elements of $\bar{\Sigma}_{p}$ set are feasible combinations for $\left\langle G_{p} ; H_{p}\right\rangle$ : $\bar{\Sigma}_{p}=\left(F_{1}, \ldots, F_{n_{p}}\right)$, where $n_{p}$ is the number of feasible combinations for $\left\langle G_{p} ; H_{p}\right\rangle$. Each feasible combination of projects $F_{j}, j=\overline{1, n_{p}}$, consists of $k$ vertices taken one from each $x_{i}, i=\overline{1, k}$. Let's denote it $\left(\xi_{j_{1}}, \ldots, \xi_{j_{k}}\right) \subset \Sigma . \bar{\Delta}$ reflection is defined in following way:

$$
\text { c) } \bar{\Delta} \mathrm{F}_{\mathrm{j}}=\bigcup_{r=1}^{k} \Delta \xi_{\mathrm{jr}} \backslash \bigcup_{i=1}^{k} \sum_{\mathrm{i}}, \quad \mathrm{j}={\overline{1, n_{\mathrm{p}}}}_{\text {; }} \text {; }
$$

d) $\bar{\Delta} \xi_{\mu}^{i}=\left\{\begin{array}{l}\Delta \xi_{\mu}^{i} \cup F_{j} \backslash \bigcup_{r=1}^{k} \Sigma_{r}, \mathrm{i}=\overline{k+1, n}, \mu=\overline{1, \mathrm{n}_{1}}, \mathrm{j}=\overline{1, \mathrm{n}_{\mathrm{p}}} \text { only in that case when } \exists \xi_{\mathrm{jr}} \text { so that } \Delta \xi_{\mu}^{i} \supset \xi_{j r}, \\ \Delta \xi_{\mu}^{i} \backslash \bigcup_{r=1}^{k} \Sigma_{r}, \mathrm{i}=\overline{k+1, n}, \mu=\overline{1, \mathrm{n}_{\mathrm{i}}}, \text { in other cases. }\end{array}\right.$ 
It is obvious, that during this process no any feasible combination may be lost or created. Following this principle, actually, for ESD GP there is some separated multitude of feasible combinations, among which the decision-maker makes his option in accordance with the chosen solution concept.

The structural graph of ESD GP includes different levels of hierarchy and it may reflect correlation between indicators of different levels of detailed elaboration. Simultaneously it enables to partially resolve problems relating to agreement of decisions between different hierarchies and reduction of calculations to select scenarios.

It is appropriate to note here that though external capital flows are not restricted and no quantitative restrictions lie on external trade transactions, their flow is, however, determined by pace of local structural reforms, risky investments, legal protection and some other factors. Armenia's industrial and financial systems still remain not differentiated, that is to say business entities still are not in a position to attract funds through the securities market. Only the secondary market of the government securities has passed a certain development in recent years. The ESD GP model considers these restrictions, which were estimated based on Armenia's 1994-2000 numerical data and time series. During this period certain rehabilitation of the economic growth has taken place, and macroeconomic stabilization and development trends have been shown.

\section{Construction of Feasible Versions on the Example of Central Bank}

Construction of the alternatives in ESD GP model is considered when supply of money is increased by 10 per cent and the behavior of the selected basic macro-indicators is provided in Table 1. In general case, feasible versions are developed for each vertex of the graph $G=(X, \tilde{A})$; for particular case, this paper reviews possible versions of implementation of the monetary policy by the Central Bank. Actually, impact of internal or external factors induces the need to develop a regulatory mechanism, which in this case is explained on the above- mentioned example. Central Bank develops relevant feasible versions in the framework of goal program, which co-integrate with feasible versions of the rest subjects (Engle and Granger, 1987). In other words, alternative versions are built also for the rest of objects included in ESD $\mathrm{GP}$, and a feasible combination in $G=(X, \tilde{A})$ is chosen in accordance with provided procedure.

Table 1. The alternative version for basic indicators due to changes in demand for money in ESD GP (deviation from the base scenario, \%)

Alternative versions of management parameters (REPO interest rate, monetary base, exchange rate, total domestic loans)

\begin{tabular}{lllll}
$\xi_{\mathrm{CB} 1}$ & $\xi_{\mathrm{CB} 2}$ & $\xi_{\mathrm{CB} 3}$ & $\xi_{\mathrm{CB} 4}$ & $\xi_{\mathrm{CB} 5}$ \\
\hline
\end{tabular}

Indicators

$\begin{array}{lccccc}\text { Inflation } & 2.86 & 3.45 & 2.45 & 6.81 & 1.23 \\ \text { GDP } & 2.15 & 3.01 & 2.02 & 3.52 & 1.85 \\ \text { REPO interest rate } & 2.67 & 3.12 & 1.85 & 3.84 & - \\ \text { Monetary base } & 0.13 & - & 8.12 & 12.54 & - \\ \text { Broad money } & 12.43 & 13.65 & 10.0 & 15.23 & 9.85 \\ \text { Exchange rate } & 3.44 & 4.72 & 3.11 & 8.50 & - \\ \text { Financial market rate } & 1.85 & 3.12 & 1.12 & 4.93 & 1.05 \\ \text { Loans to economy } & 5.59 & - & - & 6.58 & - \\ \text { Exports } & 4.31 & 4.85 & 3.76 & 4.60 & 3.54 \\ \text { Imports } & 8.75 & 9.03 & 7.68 & 8.15 & 5.50 \\ \text { Trade balance } & 14.27 & 16.45 & 12.47 & 10.24 & 11.12\end{array}$


The alternative version is provided in ESD GP based on an estimated equation of demand for money, the variants of which include the following options of the policy conduct:

i) It presents a tightly regulated option of REPO interest rate, monetary base, exchange rate, total domestic loans, to ensure the predetermined target indicators;

ii) REPO interest rate and exchange rate are accepted from parameters of management as appropriate to a level achieved in the market, while changes in volumes of monetary base and total domestic loans are constant;

iii) Changes in REPO interest rate, monetary base, exchange rate are taken as regulating, while changes in total domestic loans as constant;

iv) Changes in REPO interest rate, monetary base and total domestic loans are regulating, while exchange rate is taken as flexible;

v) Changes in all variables of management are taken as constant.

According to estimations in Table 1 , in case of growth of money supply by 10 per cent, a relatively stable level of inflation, GDP and financial market interest rate will be achieved (in case of option v). Considering that improvement of the current account through change in exchange rate is restricted due to quite strong relations between exchange rate fluctuations and the level of prices in Armenia, the current account stability, however, is achieved in option iv) in compliance with estimations produced. One accepted option is generated in case of each option of the policy conduct by the Central Bank, and another option is generated in case of impact of other internal and external factors. Generally, the possible scenarios will be reviewed in ESD GP according to various solution concepts, i.e. equilibrium, a Pareto Optimum and kernel to identify certain feasible scenarios.

Pace of implementation of economy stabilization and development programs of transition countries gives evidence that variances from determined program indicators and trends of economic development in both short and long terms emerge. To eliminate this, appropriate mechanisms will need to be developed. Unforeseeable variances from selected scenarios of economy stabilization and development will occur under expansionary monetary and fiscal policies, supply, capital flows, marketability of securities in financial market, unexpected shocks of providing loans or financial resources.

To adjust variances emerged in the outcome of evaluated correlation of medium term equilibrium and selected scenario between vertices of the structural graph, one should use the method of co-integration and the error correction model. Existence of co-integration has been revealed for equations of demand for money. Equation of demand for money covers the level of economic activity, domestic interest rates, exchange rate, and the error adjustment rate. The study of the error correction model for money demand has been carried out within a portfolio approach. According to this approach, keeping of money is seen as an alternative choice to real, financial, domestic and external assets.

Co-integrated equation of demand for money is provided as follows:

$$
\begin{gathered}
\mathrm{m}=1.15 \mathrm{y}-1.87 \mathrm{p}+2.40 \mathrm{e}-0.24 \mathrm{r} \\
(5.80) \quad(-8.34)(24.86)(-9.89) \\
\mathrm{R}^{2}=0.97 \quad \sigma=0.055 \quad \mathrm{DW}=1.82
\end{gathered}
$$

Where $\mathrm{m}, \mathrm{y}, \mathrm{p}, \mathrm{e}, \mathrm{r}$ are real demand for money, economic growth, price level, exchange rate and refinancing rate, correspondingly (logarithms of variables were used). The estimated coefficients are significant enough, however the standard errors are relatively higher. In the next phase, the error correction model was estimated, with co-integrated correlations included.

$$
\begin{aligned}
& \mathrm{dm}=0.23 * \mathrm{dm}(-2)+0.82 * \mathrm{dy}(-1)-0.51 * \mathrm{dp}(-1)+0.81 * \mathrm{de}(-1)-0.16 \mathrm{dr}(-1)- \\
& \begin{array}{llll}
(2.50) & (2.47) & (-2.13) & (2.19)
\end{array} \\
& -0.14 * \mathrm{ECM}_{\mathrm{m}}(-1)+0.17 \mathrm{D} 11+0.09 * \mathrm{D} 13-0.09 * \mathrm{D} 18 \\
& \begin{array}{llll}
(-2.59) \quad(4.61) \quad(2.53) \quad(-2.48) & (2.1)
\end{array} \\
& \mathrm{R}^{2}=0.61 \quad \sigma=0.037 \quad \mathrm{DW}=2.1
\end{aligned}
$$

The $\mathrm{ECM}_{\mathrm{m}}(-1)$ coefficient of the estimated equation (2) indicates the rate of adjusting the difference between actual and medium term equilibrium of broad money, which is around 14 percent during a 
month. t-statistics is significant on a 1 percent level of confidence. Dummies D 11, D 13 and D 18 variables are significant enough and include events and seasonal factors occurred in the policy sphere. When the level of broad money is higher than the level of the medium term equilibrium, the level of prices and the refinancing rate increases, while the demand for money decreases, according to the error correction model. The results produced from calculation of the model show that the money demand factors are concurrent with the trends shaped in other countries in transition.

\section{References}

Toroyan, V.H. and Aharonyan, A.N. (1997) "Solution Concepts in the System of Transition Period GoalOriented Programs". Proceedings of $15^{\text {th }}$ International Conference on Methods and Applications of Multiple Criteria Decision Making, Mons, (Belgium).

Engle, Robert F. and Granger, C.W.G. (1987), “ Co-Integration and Error Correction: Representation, Estimation and Testing “, Econometrics, Vol. 55, No.2, 251-276. 
